\title{
Ultrasound and multi-biomarker disease activity score for assessing and predicting clinical response to tofacitinib treatment in patients with rheumatoid arthritis
}

Amir A. Razmjou ${ }^{1}$ D, Jenny Brook², David Elashoff², Gurjit Kaeley ${ }^{3}$, Soo Choi ${ }^{4}$, Tanaz Kermani ${ }^{5}$ and Veena K. Ranganath ${ }^{*^{*}}$ D

\begin{abstract}
Background: Musculoskeletal ultrasound (MSUS) and the multi-biomarker disease activity (MBDA) score are outcome measures that may aid in the management of rheumatoid arthritis (RA) patients. This study evaluated tofacitinib response by MSUS/MBDA scores and assessed whether baseline MSUS/MBDA scores or their early changes predict later clinical response.

Methods: Twenty-five RA patients treated with tofacitinib were assessed at baseline, 2, 6 and 12-weeks. Power doppler (PDUS) and gray scale (GSUS) ultrasound scores, MBDA score, clinical disease activity index (CDAl), and disease activity score (DAS28) were obtained. Pearson correlations and multiple linear regression models were used to evaluate associations and identify predictors of response to therapy.

Results: MSUS, MBDA scores, CDAI, and DAS28 improved significantly over 12 weeks $(p<0.0001)$. Baseline MSUS and MBDA score correlated with each other, and with 12-week changes in CDAI/DAS28 $(r=0.45-0.62, p<0.05)$, except for GSUS with DAS28. Two-week change in MSUS correlated significantly with 12-week changes in CDAI/ DAS28 ( $r=0.42-0.57, p<0.05)$, except for early change in PDUS with 12-week change in CDAl. Regression analysis demonstrated significant independent associations between baseline PDUS/MBDA score and 6-week change in CDAI/DAS28, with adjustment for baseline CDAI/DAS28 (all $p<0.05$ ); and between baseline MBDA scores and 12week change in DAS28 $(p=0.03)$.
\end{abstract}

Conclusions: RA patients treated with tofacitinib for 12 weeks demonstrated improvement by clinical, imaging, and biomarker end-points. Baseline PDUS and MBDA score were predictive of CDAI and DAS28 responses. This is the first study to evaluate early measurements of MSUS and MBDA score as predictors of clinical response in RA patients treated with tofacitinib.

Trial registration: ClinicalTrials.gov NCT02321930 (registered 12/22/2014).

Keywords: Rheumatoid arthritis, Ultrasound, Outcome measures, Multi-biomarker disease activity score

* Correspondence: vranganath@mednet.ucla.edu

${ }^{5}$ Department of Rheumatology, UCLA-David Geffen School of Medicine, 1000 Veteran Blvd., RM 32-59, Los Angeles, CA 90095, USA

Full list of author information is available at the end of the article

(c) The Author(s). 2020 Open Access This article is licensed under a Creative Commons Attribution 4.0 International License, which permits use, sharing, adaptation, distribution and reproduction in any medium or format, as long as you give appropriate credit to the original author(s) and the source, provide a link to the Creative Commons licence, and indicate if changes were made. The images or other third party material in this article are included in the article's Creative Commons licence, unless indicated otherwise in a credit line to the material. If material is not included in the article's Creative Commons licence and your intended use is not permitted by statutory regulation or exceeds the permitted use, you will need to obtain permission directly from the copyright holder. To view a copy of this licence, visit http://creativecommons.org/licenses/by/4.0/ The Creative Commons Public Domain Dedication waiver (http://creativecommons.org/publicdomain/zero/1.0/) applies to the data made available in this article, unless otherwise stated in a credit line to the data. 


\section{Background}

Early treatment and vigilant monitoring are important for preventing joint destruction, impaired function, and poor quality of life in patients with rheumatoid arthritis (RA). Numerous metrics are available to monitor disease activity and inform treatment decisions. Validated RA outcomes include questionnaire-based metrics (Routine Assessment of Patient Index Data 3 [RAPID-3]; Health Assessment Questionnaire [HAQ]), clinical metrics (Clinical Disease Activity Index [CDAI]) and composites of clinical and laboratory-based metrics (e.g., Disease Activity Score [DAS]). While these measures have advanced RA patient care over the last few decades, concerns regarding their subjectivity indicate that objective, sensitive measures are still needed $[1,2]$.

Musculoskeletal ultrasound (MSUS) and the multibiomarker disease activity (MBDA) scores are measures that can aid in the management of RA patients. These measures are responsive to RA treatment and may provide additional information beyond that of standard disease activity RA metrics [3, 4]. MSUS assesses synovitis by power Doppler (PDUS) and gray-scale (GSUS) and has been deemed appropriate for use in the management of RA by the American College of Rheumatology (ACR) [5] and the European League Against Rheumatism (EULAR) [3]. MSUS is often more sensitive for detecting synovitis than physical exam alone $[6,7]$, and has been shown to predict flares of RA $[8,9]$. The MBDA score is a validated blood test that measures 12 serum biomarkers for an algorithm that scores RA disease activity on a scale of 1-100. The MBDA score correlates with DAS28-CRP and is a predictor of risk for radiographic progression $[10,11]$.

Tofacitinib is a small-molecule oral Janus kinase (JAK) inhibitor that is approved for treatment of RA [12]. The efficacy and safety of tofacitinib $5 \mathrm{mg}$ twice daily, with or without conventional synthetic disease modifying antirheumatic drugs (csDMARDs), has been demonstrated in Phase 2 and Phase 3 studies of patients with RA [13-16]. No published studies have assessed MSUS or the MBDA score in RA patients treated with tofacitinib.

Examining the relationship between early changes in MSUS/MBDA scores to later changes in DAS/CDAI is of interest, as it may potentially allow for earlier identification of individuals who benefit from therapy. Additionally, baseline values of MSUS/MBDA scores may aid in differentiating inflammatory disease amenable to treatment, in RA patients with comorbidities where components of DAS/CDAI may be falsely elevated [1, 17]. This study evaluated the response of MSUS (PDUS and GSUS) and the MBDA score in patients treated with tofacitinib, and assessed whether baseline MSUS and MBDA scores or their early changes are predictive of later clinical response as measured by CDAI and DAS28.

\section{Methods}

\section{Setting}

This study was a single-center, open-label, single-arm trial of RA patients treated with oral tofacitinib. The study was conducted at the University of California, Los Angeles (UCLA) Rheumatology Clinics between February 2016 and August 2017. All participants provided written informed consent in person, prior to the conduct of any study procedures. The study was reviewed by the Institutional Review Board (IRB) (IRB\# 14-001148) and registered with clinicaltrials.gov (NCT02321930, ID: WI193025).

\section{Subjects}

Inclusion criteria included meeting the ACR/EULAR 2010 RA classification criteria [18], baseline DAS28/ $E S R \geq 3.2$, baseline total PDUS score $\geq 10$ (see below for scoring system), age $\geq 18$, and stable ongoing treatment with a conventional synthetic DMARD (csDMARD), such as methotrexate, leflunomide, hydroxychloroquine, sulfasalazine. Exclusion criteria included current use of prednisone $>10 \mathrm{mg}$ per day, prior treatment with tofacitinib, concomitant biologic therapy, active infection, untreated latent tuberculosis, or significant organ dysfunction.

\section{Study design, treatment, and clinical and laboratory assessments}

Study participants were treated with tofacitinib $5 \mathrm{mg}$ orally twice a day for a total of 12 weeks. Patient demographics were collected at the screening visit prior to treatment. Patients were assessed at baseline, 2 weeks, 6 weeks, and 12 weeks. At each visit, patients were assessed by the tender joint count 28 (TJC28), swollen joint count 28 (SJC28), acute phase reactants (high-sensitivity Creactive protein [hsCRP], erythrocyte sedimentation rate [ESR]), physician and patient global assessments and HAQ. CDAI and DAS28-ESR (DAS28) were determined for each visit. Rheumatoid factor (RF) and anti-cyclic citrullinated peptide antibody (ACPA) were obtained at baseline.

\section{Musculoskeletal ultrasound}

MSUS assessments (PDUS and GSUS) were obtained at screening, baseline, 2 weeks, and 12 weeks by the same sonographer, who had greater than 8 years of experience and is ACR certified in MSUS (RhMSUS certification) (VKR). MSUS assessment was blinded to patient clinical data. A 34-joint US scoring system was used, which assessed bilateral midline wrists (dorsal longitudinal), radio-ulnar joints (dorsal longitudinal/short midline), metacarpophalangeal joints 1-5 (MTP, dorsal long and short, volar longitudinal), proximal interphalangeal joints 2-5 (dorsal/volar longitudinal), metatarsophalangeal joints 2-5 (dorsal longitudinal), and knees (medial 
parapatellar/lateral parapatellar axial oblique). The MSUS 34-joints were chosen based on commonly affected joints in RA and feasibility of acquiring images, and this protocol has been previously used $[1,19,20]$. MTP1 was excluded as this joint is commonly affected by osteoarthritis. Each image was scored semi-quantitatively on a scale of $0-3$ for both PDUS and GSUS. We utilized a modification of the PDUS semiquantitative scoring system proposed by Hammer et al.: $0=$ none, $1=$ minor, $2=$ moderate, and $3=$ major degree of PDUS activity $[20,21]$. We created an atlas that iteratively improved on the helpful Hammer atlas to allow for improved reliability scores [20]. Each image was also scored for GSUS in the following manner: no synovitis $=$ grade 0 , minor synovitis $=$ grade 1 , moderate synovitis $=$ grade 2 , major synovitis $=$ grade 3 . Total PDUS and GSUS scores were calculated as follows: If multiple views of a single joint were assessed, the view with the maximum score was used for the joint, and these 34 scores were summed to represent the total PDUS or GSUS score (range 0-102) [1, 20]. Hereinafter, the total 34-joint PDUS score will be referred to as PDUS, and similarly the total 34 -joint GSUS score will be GSUS. A modified version of the German ultrasound 7 score (mUS7) for PDUS and GSUS was also calculated [22]. The mUS7 PDUS and mUS7 GSUS scores do not include a palmar wrist view. Thus, the range of mUS7 PDUS is 036 (rather than 0-39), and the range of mUS7 GSUS is 024 (rather than 0-27). The same ultrasound machine and transducer (a GE Logic E9 with a 6-15 MHz linear probe; GE Healthcare, Chicago, IL) and standardized settings (red-yellow color map, Doppler frequency: $10.0 \mathrm{MHz}$, PRF $0.8 \mathrm{KHz}$, gain adjusted just below noise) were used for each visit and joint for all patients. The same transducer was used for the finger and the knee parapatellar recesses, however, adjustments were made for depth. Ten percent of the images were rescored and compared to the original score. The intra-rater reliability was 0.82 for PDUS and 0.76 for GSUS (weighted Kappa).

\section{MBDA testing}

MBDA scores were determined for all patients at baseline, week 2 and week 12 from serum samples that were shipped on ice within few hours of phlebotomy to the CLIA-certified clinical laboratory of Crescendo Bioscience, Inc., in South San Francisco, CA, for testing. MBDA scores were determined with a validated algorithm based on serum concentrations of 12 protein biomarkers [10]. The biomarkers in the MBDA test reflect the biology of RA and consist of cytokine-related proteins (IL-6, TNF-R1), acute phase reactants (CRP, serum amyloid A), an adhesion molecule (VCAM-1), growth factors (EGF, VEGF-A), matrix metalloproteinases (MMP-1, MMP-3), and adipokines (leptin, resistin). The MBDA score is an integer on a scale of 1 to 100, with disease activity categories of low $(<30)$, moderate $(30$ 44) and high (>44) [10]. Minimally clinically important difference (MCID) for MBDA score is $\geq 8$ [23].

\section{Statistical analysis}

The sample size for the study was calculated for the primary analysis of comparing PDUS between baseline and week 12. Based on unpublished data suggesting an effect size for change in PDUS over time of 1.03 (unpublished data), the planned sample size of 25 had $99 \%$ power for detecting differences in PDUS over 3 months, assuming a repeated measures ANOVA analysis plan with a 0.05 two-sided level of significance. Repeated measures ANOVA models were used to evaluate changes over time in MSUS, MBDA score and RA disease activity measurements. Each variable was examined for skewness and not found to be substantially skewed. Thus, Pearson correlations were computed for associations between MSUS or MBDA scores and CDAI or DAS28, which were evaluated crosssectionally and as changes over time. In addition, correlations were calculated between MSUS/MBDA scores and individual components of DAS28/CDAI. Multiple linear regression models for the outcomes of change in CDAI and change in DAS28 were constructed. These models included baseline MSUS or MBDA scores, adjusting for baseline CDAI and DAS28 as appropriate.

\section{Results}

Twenty-five RA patients were enrolled in the trial. Mean age was 52 (SD 9.9) years, mean duration of disease 10.4 years (SD 9.7); $88 \%$ of patients were female, $40 \%$ Caucasian, $88 \%$ were RF and/or ACPA positive (Table 1). At baseline, $72 \%$ of patients were receiving a csDMARD, most commonly methotrexate (52\%), and 56\% were biologic naïve.

\section{Ultrasound, MBDA score, and RA disease activity measures over 12 weeks}

At baseline, patients had high disease activity with a mean CDAI of 39.9 (SD 13.2) and mean DAS28 of 6.3 (SD 1.2) (Table 2). The baseline mean MBDA score was 50.6 (SD 17.5), mean PDUS 28.7 (SD 17.8), mean GSUS was 48.4 (SD 16.5), mean mUS7 PDUS was 10.2 (SD 6.5), and mean mUS7 GSUS was 18.0 (7.1) (Fig. 1). In response to tofacitinib, all of these indices decreased significantly over the 12-week follow-up $(p<0.0001)$ (Table $2)$. The standardized response means (SRM) for PDUS and mUS7 PDUS (1.18 and 1.10), and GSUS and mUS7 GSUS (0.90 and 0.95) were similar. The MBDA score decreased from baseline to 12 weeks by $\geq 8$ units (i.e., the minimally important difference), in 12 (50\%) patients. Twelve-week changes in DAS28/CDAI resulted in the largest standardized response means (SRM) (1.71-1.73), while hsCRP had the smallest SRM (0.36). 
Table 1 Baseline Patient Characteristics

\begin{tabular}{|c|c|}
\hline Total Number of Patients & 25 \\
\hline Age (years), mean (SD) & $52(9.9)$ \\
\hline Females (percent) & $22(88 \%)$ \\
\hline \multicolumn{2}{|l|}{ Race n (\%) } \\
\hline Caucasian & $10(40 \%)$ \\
\hline Black & $4(16 \%)$ \\
\hline Hispanic & $5(20 \%)$ \\
\hline Asian & $4(16 \%)$ \\
\hline Other & $2(8 \%)$ \\
\hline Seropositive n (\%) & $22(88 \%)$ \\
\hline Disease Duration (years), mean (SD) & $10.4(9.7)$ \\
\hline BMI, mean (SD) & $31.0(10.8)$ \\
\hline Prednisone at Baseline $n(\%)$ & $7(28 \%)$ \\
\hline csDMARDs at Baseline $\mathrm{n}(\%)$ & $18(72 \%)$ \\
\hline Methotrexate & $13(52 \%)$ \\
\hline Sulfasalazine & $4(16 \%)$ \\
\hline Hydroxychloroquine & $4(16 \%)$ \\
\hline Leflunomide & $4(16 \%)$ \\
\hline \multicolumn{2}{|l|}{ bDMARDs before Baseline $\mathrm{n}(\%)$} \\
\hline TNF inhibitor & $8(32 \%)$ \\
\hline IL-6 inhibitor & $1(4 \%)$ \\
\hline Anti-CTLA4-lgG & $2(8 \%)$ \\
\hline None of the Above & $14(56 \%)$ \\
\hline Comorbidities (number), mean (SD) & $3.8(2.6)$ \\
\hline
\end{tabular}

SD Standard Deviation, RF Rheumatoid Factor, ACPA Anti-Citrullinated Protein Antibody; BMI = Body Mass Index; $\operatorname{csDMARDs}=$ Conventional Synthetic Disease Modifying Anti-Rheumatic Drugs; bDMARDs = Biological DMARDs

\section{Relationships between ultrasound and MBDA scores}

MBDA score demonstrated significant cross-sectional correlations with PDUS at baseline $(\mathrm{r}=0.74, p<0.01), 2$ weeks $(\mathrm{r}=0.61, p<0.05)$ and 12 weeks $(\mathrm{r}=0.50, p<$ $0.05)$; and with GSUS at baseline $(\mathrm{r}=0.61, p<0.01)$ and 2 weeks $(\mathrm{r}=0.41, p<0.05)$ but not 12 weeks $(\mathrm{r}=0.22$, $p>0.05)$. These results show that correlations between MBDA score and ultrasound measures were strongest at baseline and remained statistically significant during treatment with tofacitinib.

\section{Cross-sectional correlations between ultrasound, MBDA} score, and RA disease activity outcomes

Cross-sectional analyses demonstrated that PDUS and GSUS correlated significantly with CDAI and DAS28 at all study time points (baseline, 2 weeks and 12 weeks) (Pearson correlations ranging from $r=0.45$ to $r=0.65$, $p<0.05)$, with the exception of 12-week PDUS with 12week CDAI $(r=0.38, p>0.05)$ (Table 3). Both mUS7 PDUS and mUS7 GSUS demonstrated similar correlation with CDAI and DAS28 $(\mathrm{r}=0.41-0.58, p<0.05)$ with the exception of 2-week mUS7 GSUS with 2-week
DAS28, and 12-week mUS7 PDUS with 12-week CDAI. The magnitude of these correlations generally decreased over time. MBDA score correlated significantly with DAS28 at baseline $(r=0.47, p<0.05)$, but not at weeks 2 or 12 , and it did not correlate significantly with CDAI at any time point.

\section{Relationships between 12-week changes in ultrasound,} MBDA score and CDAI and DAS28

Changes in MSUS measures and the MBDA score from baseline to 12 weeks all correlated significantly with changes from baseline to 12 weeks in CDAI and DAS28 (range: $\mathrm{r}=0.44$ to $\mathrm{r}=0.64, p<0.05$ ), with the exception of 12-week changes in GSUS and DAS28 (Table 3).

\section{Relationships between early measurements of ultrasound/} MBDA score and later responses in CDAI/DAS28

Baseline MSUS measures and the MBDA score correlated significantly with changes in CDAI and DAS28 from baseline to week 12 (range: $\mathrm{r}=0.45$ to $\mathrm{r}=0.55, p<0.05$ ), with the exception of baseline GSUS and mUS7 GSUS with 12week change in DAS28 (Table 3). Changes in MSUS measures from baseline to week 2 correlated significantly with changes from baseline to week 12 in CDAI and DAS28 (range of $r=0.42$ to $r=0.44, p<0.05$ ), with the exception of 2-week change in PDUS with 12-week change in CDAI (Table 3). Change in MBDA score from baseline to week 2 did not correlate significantly with 12 -week changes in CDAI or DAS28.

Relationships between ultrasound, MBDA score, and individual components of CDAI and DAS28 (TJC28, SJC28, MD global, patient global, ESR)

Cross-sectionally, MSUS measures correlated with SJC28 at baseline, and week 2 ( $\mathrm{r}=0.43$ to $0.76, p<0.05$ ). MSUS measures and the MBDA score correlated well with ESR at baseline ( $r=0.50$ to $0.74, p<0.05$ ). None of the MSUS measures or the MBDA score correlated with TJC28 at any time point.

\section{Multiple linear regression for associations between baseline ultrasound score and changes in CDAI and DAS28 from baseline to weeks 6 or 12}

Multiple linear regression demonstrated a positive independent association between baseline PDUS and change in DAS28 from baseline to week $6(p=0.01)$, with adjustment for baseline DAS28. Similarly, baseline PDUS was independently associated with change in CDAI from baseline to week 6 ( $p=0.004)$, with adjustment for baseline CDAI. Baseline PDUS showed a trend toward positive association with changes in CDAI and DAS28 from baseline to week 12, ( $p=0.07$ and $p=0.05$, respectively). By contrast, GSUS was not associated with CDAI or with DAS28 responses from baseline to week 6 or week 
Table 2 Ultrasound, MBDA Score, and RA Disease Activity Measures Over 12 Weeks

\begin{tabular}{|c|c|c|c|c|c|}
\hline Measure (range/unit) & $\begin{array}{l}\text { Baseline mean (SD) } \\
N=25\end{array}$ & $\begin{array}{l}2 \text { weeks mean (SD) } \\
N=25\end{array}$ & $\begin{array}{l}12 \text { weeks mean (SD) } \\
N=24\end{array}$ & $p$-value & SRM \\
\hline PDUS (0-102) & $28.7(17.8)$ & $19.5(15.4)$ & $12.2(10.6)$ & $<0.0001$ & 1.18 \\
\hline GSUS (0-102) & $48.4(16.5)$ & $44.9(16.4)$ & $37.9(15.3)$ & $<0.0001$ & 0.90 \\
\hline mUS7 PDUS (0-36) & $10.2(6.5)$ & $7.0(5.2)$ & $4.1(4.0)$ & $<0.0001$ & 1.10 \\
\hline mUS7 GSUS (0-24) & $18.0(7.1)$ & $15.7(7.1)$ & $12.9(5.9)$ & $<0.0001$ & 0.95 \\
\hline MBDA Score (1-100) & $50.6(17.5)$ & $41.0(15.1)$ & $39.6(15.3)$ & $<0.0001$ & 0.73 \\
\hline CDAI (0-76) & $39.9(13.2)$ & $28.6(13.2)$ & $21.6(13.0)$ & $<0.0001$ & 1.73 \\
\hline DAS28/ESR (0- 9.1) & $6.3(1.2)$ & $5.2(1.3)$ & $4.6(1.4)$ & $<0.0001$ & 1.71 \\
\hline TJC28 (0-28) & $12.6(6.5)$ & $8.7(6.5)$ & $6.3(5.8)$ & $<0.0001$ & 1.12 \\
\hline SJC28 (0-28) & $13.0(6.4)$ & $9.3(6.1)$ & $7.1(5.4)$ & $<0.0001$ & 1.26 \\
\hline Physician Global (0-10) & $6.8(2.0)$ & $5.4(1.6)$ & $4.2(1.6)$ & $<0.0001$ & 1.53 \\
\hline Patient Global (0-10) & $7.0(1.9)$ & $4.9(2.1)$ & $3.5(2.6)$ & $<0.0001$ & 1.31 \\
\hline $\operatorname{ESR}(\mathrm{mm} / \mathrm{hr})$ & $39.2(27.4)$ & $31.6(22.0)$ & $27.6(20.3)$ & 0.003 & 0.55 \\
\hline hsCRP (mg/L) & $16.7(20.8)$ & $6.7(7.4)$ & $8.1(16.7)$ & 0.02 & 0.36 \\
\hline HAQ-DI (0-3) & $1.4(0.7)$ & $1.0(0.6)$ & $0.7(0.6)$ & $<0.0001$ & 0.83 \\
\hline
\end{tabular}

PDUS Power doppler ultrasound, GSUS Gray scale ultrasound, mUS7 Modified ultrasound 7, MBDA Multi-biomarker disease activity, CDAI Clinical disease activity index, DAS28/ESR Disease activity score, erythrocyte sedimentation rate, TJC Tender joint count, SJC Swollen joint count, hsCRP High-sensitivity C-reactive protein, $H A Q-D I$ Health assessment questionnaire-disability Index, SRM Standardized Response Means. $P$-values represent the significance of the effect of time in repeated measures ANOVA models

12. The baseline CDAI and baseline DAS28 were not significantly associated with the 6-week or 12-week responses to therapy for CDAI or DAS28.

\section{Multiple linear regression for associations between baseline MBDA score and 6-week and 12-week changes in CDAl and DAS28}

Multiple linear regression demonstrated a positive independent association between baseline MBDA score and change in CDAI or DAS28 from baseline to week 6 (both $p=0.006$ ), with adjustment for baseline CDAI and DAS28, respectively. Baseline MBDA score was associated with 12 -week change in DAS28 $(p=0.03)$, with adjustment for baseline DAS28, with a trend toward association with 12-week change in CDAI $(p=0.08)$, with adjustment for baseline CDAI.

\section{Safety}

There were two reports of adverse events, both moderate in severity. One patient had a tendon rupture of

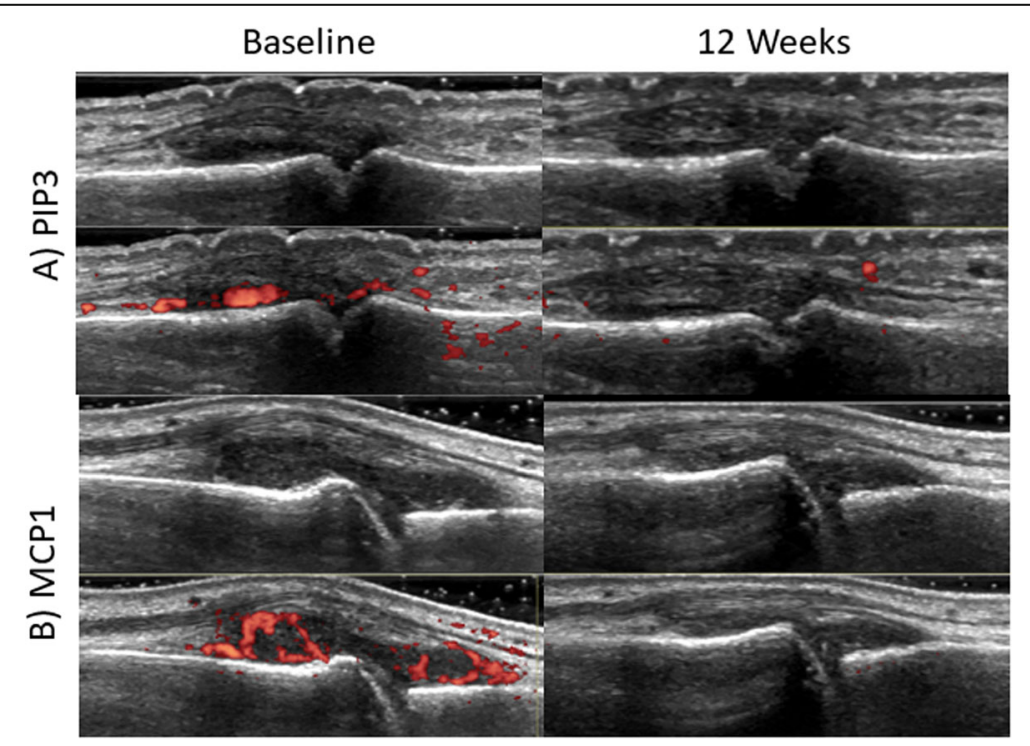

Fig. 1 Baseline and 12-Week MSUS images after treatment with tofacitinib (GSUS and PDUS). Panels $\mathbf{a}$ and $\mathbf{b}$ show images of PIP3 and MCP1 in both B-mode and power Doppler before and after treatment with tofacitinib 
Table 3 Correlations between MSUS/MBDA Score and Clinical Disease Activity Measures

Baseline MSUS/MBDA score association with Baseline CDAI/DAS28/ESR

PDUS Baseline

\section{$0.53^{* *}$}

GSUS Baseline

mUS7 PDUS Baseline

mUS7 GSUS Baseline

MBDA Score Baseline

2 Week MSUS/MBDA score association with 2 Week CDAI/DAS28

\section{CDAl 2 Week}

PDUS 2 Week

GSUS 2 Week

mUS7 PDUS 2 Week

mUS7 GSUS 2 Week

MBDA Score 2 Week
CDAI Baseline

DAS28/ESR Baseline
$0.58^{* *}$
$0.53^{* *}$
$0.58^{* *}$
$0.42^{*}$
$0.47^{*}$

DAS28/ESR 2 Week

$0.49^{*}$

$0.52^{* *}$

$0.49^{*}$

0.31

0.19

\section{DAS28/ESR 12 Week}

$0.54^{* *}$

$0.53^{* *}$

$0.43^{*}$

$0.44^{*}$

0.05

DAS28/ESR ( $\Delta$ 0-12 weeks)

$0.45^{*}$

0.25

$0.55^{* *}$

0.33

$0.50^{*}$

DAS28/ESR ( $\Delta$ 0-12 weeks)

$0.42^{*}$

$0.42^{*}$

$0.57^{* *}$

$0.51^{* *}$

$0.22 \quad 0.29$

12 Week change in MSUS/MBDA score association with 12 Week change in CDAI/DAS28

CDAl ( $\triangle$ 0-12 week)

DAS28/ESR ( $\triangle$ 0-12 week)

PDUS ( $\triangle$ 0-12 week)

$0.58^{* *}$

$0.56^{* *}$

$0.47^{*}$

0.34

$0.64^{* *}$

$0.60^{* *}$

$0.48^{*}$

mUS7 GSUS ( $\Delta$ 0-12 week)

$0.54^{* *}$

$0.59^{* *}$

${ }^{*} p<0.05,{ }^{*} p<0.01$; PDUS Power doppler ultrasound, GSUS Gray scale ultrasound, mUS7 modified ultrasound 7, MBDA Multi-biomarker Disease activity score, CDAI Clinical disease activity index, DAS28/ESR Disease activity score/erythrocyte sedimentation rate 
unclear etiology and the other had an upper respiratory infection. Another patient was withdrawn from the study after the week 2 visit, due to the development of a breast mass during the trial, with subsequent imaging demonstrating a simple cyst. In addition, one patient was diagnosed with diffuse large B-cell lymphoma approximately 2 months after conclusion of the trial.

\section{Discussion}

This study investigated the response of RA patients treated with tofacitinib using traditional clinical measures (CDAI and DAS28), and imaging and biomarker measures (MSUS and MBDA score). Our data is consistent with other studies of tofacitinib which demonstrated significant responses by CDAI and DAS28 $[15,16,24]$ in patients with RA. This study is the first to demonstrate significant improvements in MSUS measures (with both 34-joint, and mUS7 methods)and MBDA scores in response to tofacitinib. Moreover, it demonstrated that PDUS and MBDA score were correlated with each other and that, at baseline, each predicted later responses of CDAI and DAS28.

Several studies have demonstrated the ability of MSUS to monitor treatment response and predict radiographic progression in patients with RA, including patients in clinical remission [9, 25-28]. Fewer studies have evaluated baseline MSUS and its early changes for predicting later clinical response to treatment [29-31]. Kawashiri and colleagues evaluated 39 RA patients treated with biologic DMARDs over 24 weeks. They found that the percentage decrease of GSUS and PDUS over 12 weeks was greater for those patients with EULAR moderate and good responses compared to non-responders at 24 weeks $(p<0.05)$ [31]. Ellegaard et al. demonstrated in a cohort of 109 RA patients that baseline PDUS predicted persistence of anti-TNF-alpha therapy whereas conventional clinical measures at baseline did not [29]. In a study of 25 biologic-naïve RA patients treated with abatacept, we previously found that baseline PDUS, measured using the US7 ultrasound protocol [22], significantly correlated with 52-week change in DAS28 [30]. In addition, there was a trend toward the association of PDUS with 52-week change in DAS28 after adjustment for baseline DAS28 (0.06).

The present tofacitinib study found that higher baseline PDUS values were associated with larger CDAI and DAS28 responses at 6 weeks, with a trend observed at 12 weeks. Our study is distinct among imaging studies in that we included baseline clinical disease activity measures in the multiple regression analyses. Additionally, we found that early changes in PDUS,GSUS, and mUS7 scores, i.e., from baseline to 2 weeks, showed a significant correlation with 12-week changes in CDAI and DAS28.
Prior studies have reported that baseline MBDA score can predict risk for radiographic progression [11, 3234], and that the MBDA score is a better predictor of radiographic progression than conventional measures of RA disease activity [33, 35]. A study of patients with early RA found that lower MBDA scores in patients with inadequate clinical response to methotrexate monotherapy were associated with better clinical responses to subsequent triple therapy [36]. Another study of patients with early RA - treated with MTX alone or with adalimumab - found that, for each treatment, baseline MBDA score and change in MBDA score from baseline to 3 months were associated with DAS28 improvement from baseline to 6 months [31]. Our study, of tofacitinib treatment for patients with established RA, showed that baseline MBDA score correlated significantly with changes in CDAI and DAS28 from baseline to week 12, although change in MBDA score, from baseline to week 2, did not correlate with the later changes. The MBDA score significantly correlated with PDUS at all timepoints, with the strongest correlation found at baseline $(r=0.74)$, suggesting that there may be biologic differences in therapeutic responsiveness of these measures.

The MSUS and MBDA scores, at baseline and their early changes, may be of clinical value for predicting outcomes in patients started on treatment with tofacitinib. In this RA cohort with high disease activity, MSUS/ MBDA scores correlated fairly well with the clinical measures of CDAI/DAS28. In a more heterogeneous population (e.g., with obesity, hand osteoarthritis, fibromyalgia), PDUS and MBDA score may help in the assessment of RA disease activity and stratify patients according to predicted response. If baseline measures or their early changes suggest possible treatment failure, an earlier change of treatment could be considered, potentially improving the clinical course and minimizing unnecessary healthcare utilization. In addition, elevated baseline MSUS and/or MBDA scores may provide value as inclusion criteria in randomized clinical trials allowing for a more homogenous group of RA patients with high disease activity and risk for joint damage [37].

Interestingly, when assessing the correlations of MSUS measures and the MBDA score with the individual components of the CDAI and DAS28 (TJC28, SJC28, MD Global, Patient Global, ESR), we found no significant correlations with TJC28 across any time point. In their review, Edwards et al. found that pain catastrophizing, or as they defined "the tendency to ruminate about and magnify pain", and depression were frequently observed in patients with rheumatologic conditions, and adversely affect several outcomes [38]. In a prospective trial of RA patients starting bDMARDs, Hammer et al. found that pain catastrophizing was associated with patient reported outcomes and the disease activity scores which 
incorporated them; however, there was no correlation with MSUS [2]. Data such as this suggests a complex interplay between the perception of pain, patient reported outcomes, and inflammatory disease. Further research is warranted.

Limitations of this study pertain to its sample size of twenty-five patients. Nevertheless, we did demonstrate significance in the primary end-points. The small sample size limited our ability to add other covariates into the linear regression model. The patient cohort had high baseline clinical disease activity (CDAI 39.9, DAS28 6.23), as well as long disease duration (10.4 years), which may limit the applicability of the data to other patient populations.

\section{Conclusions}

The results of this open-label trial suggest that baseline PDUS and MBDA scores correlated with each other, tracked response to treatment with tofacitinib and, at baseline, predicted 6-week and 12-week clinical responses to therapy. In addition, the 2-week change in PDUS was found to be predictive of 12-week DAS28 response. Future studies could aim to further elucidate the utility of MSUS and the MBDA score in RA management, and investigate how early changes in these measures may help provide individualized RA patient care.

\section{Abbreviations}

RA: Rheumatoid Arthritis; RAPID-3: Routine Assessment of Patient Index Data 3; HAQ: Health Assessment Questionnaire; CDAl: Clinical Disease Activity Index; DAS: Disease Activity Score; MSUS: Musculoskeletal Ultrasound; MBDA: Multi-biomarker Disease Activity; PDUS: Power Doppler Ultrasound; GSUS: Gray Scale Ultrasound; ACR: American College of Rheumatology; EULAR: European League Against Rheumatism; csDMARDs: Conventional Synthetic Disease Modifying Antirheumatic Drugs; TJC28: Tender Joint Count 28; SJC28: Swollen Joint Count 28; hsCRP: High-sensitivity C-reactive Protein; ESR: Erythrocyte Sedimentation Rate; RF: Rheumatoid Factor; ACPA: Anticyclic Citrullinated Peptide Antibody; MCID: Minimal Clinically Important Difference

\section{Acknowledgements}

Not applicable.

\section{Conflict of interest}

AA Razmjou, MD- None, J Brook, MS- None, G Kaeley, MD- None, D Elashoff, PhD- None, S Choi, MD- None, TA Kermani, MD- None, VK Ranganath, MD, MS- consultant BMS, DSMB Amgen, grants- Pfizer, BMS, Mallinckrodt, Genentech.

\section{Authors' contributions}

Study design- VKR, GK, and DAE. Data acquisition: JB, VKR, SC, and TK. Data analysis and interpretation: AAR, GSK, JB, SC, TK, DAE, and VKR. Drafting manuscript: AAR, GSK, JB, SC, TK, DAE, and VKR. Final version approval: AAR, GSK, JB, SC, TK, DAE, and VKR.

\section{Funding}

Funding for this investigator-initiated trial was provided by Pfizer.

Investigator initiated trial funded by Pfizer.

\section{Availability of data and materials}

The datasets used and/or analyzed during the current study are available from the corresponding author on reasonable request.

\section{Ethics approval and consent to participate}

Ethics approval by the Institutional Review Board (IRB) (IRB\# 14-001148). Consent provided by each participating subject in the study.

\section{Consent for publication}

Not applicable.

\section{Competing interests}

VK Ranganath, MD, MS- consultant BMS, DSMB Amgen, grants- Pfizer, BMS, Mallinckrodt, Genentech. All other authors have no competing interests to declare.

\section{Author details}

${ }^{1}$ Department of Medicine, UCLA-David Geffen School of Medicine, Los Angeles, USA. ${ }^{2}$ Department of Medicine Statistics Core, UCLA-David Geffen School of Medicine, Los Angeles, USA. ${ }^{3}$ Department of Rheumatology, University of Florida Health, Jacksonville, USA. ${ }^{4}$ Department of Rheumatology, University of California, San Diego, USA. ${ }^{5}$ Department of Rheumatology, UCLA-David Geffen School of Medicine, 1000 Veteran Blvd., RM 32-59, Los Angeles, CA 90095, USA.

Received: 6 April 2020 Accepted: 5 August 2020

Published online: 19 October 2020

\section{References}

1. Bauer EM, Ben-Artzi A, Duffy EL, Elashoff DA, Vangala SS, Fitzgerald J, et al. Joint-specific assessment of swelling and power Doppler in obese rheumatoid arthritis patients. BMC Musculoskelet Disord. 2017;18(1):99.

2. Hammer HB, Uhlig T, Kvien TK, Lampa J. Pain catastrophizing, subjective outcomes, and inflammatory assessments including ultrasound: results from a longitudinal study of rheumatoid arthritis patients. Arthritis Care Res (Hoboken). 2018;70(5):703-12.

3. Colebatch AN, Edwards CJ, Ostergaard M, van der Heijde D, Balint PV, D'Agostino MA, et al. EULAR recommendations for the use of imaging of the joints in the clinical management of rheumatoid arthritis. Ann Rheum Dis. 2013;72(6):804-14.

4. Segurado OG, Sasso EH. Vectra DA for the objective measurement of disease activity in patients with rheumatoid arthritis. Clin Exp Rheumatol. 2014;32(5 Suppl 85):S-29-34.

5. McAlindon T, Kissin E, Nazarian L, Ranganath V, Prakash S, Taylor M, et al. American College of Rheumatology report on reasonable use of musculoskeletal ultrasonography in rheumatology clinical practice. Arthritis Care Res (Hoboken). 2012;64(11):1625-40.

6. Karim Z, Wakefield RJ, Quinn M, Conaghan PG, Brown AK, Veale DJ, et al. Validation and reproducibility of ultrasonography in the detection of synovitis in the knee: a comparison with arthroscopy and clinical examination. Arthritis Rheum. 2004;50(2):387-94.

7. Szkudlarek M, Court-Payen M, Jacobsen S, Klarlund M, Thomsen HS, Ostergaard M. Interobserver agreement in ultrasonography of the finger and toe joints in rheumatoid arthritis. Arthritis Rheum. 2003;48(4):955-62.

8. Naredo E, Valor L, De la Torre I, Montoro M, Bello N, Martinez-Barrio J, et al. Predictive value of Doppler ultrasound-detected synovitis in relation to failed tapering of biologic therapy in patients with rheumatoid arthritis. Rheumatology (Oxford). 2015;54(8):1408-14.

9. Nguyen H, Ruyssen-Witrand A, Gandjbakhch F, Constantin A, Foltz V, Cantagrel A. Prevalence of ultrasound-detected residual synovitis and risk of relapse and structural progression in rheumatoid arthritis patients in clinical remission: a systematic review and meta-analysis. Rheumatology (Oxford). 2014;53(11):2110-8.

10. Curtis JR, van der Helm-van Mil AH, Knevel R, Huizinga TW, Haney DJ, Shen $Y$, et al. Validation of a novel multibiomarker test to assess rheumatoid arthritis disease activity. Arthritis Care Res (Hoboken). 2012;64(12):1794-803.

11. Hambardzumyan K, Bolce R, Saevarsdottir S, Cruickshank SE, Sasso EH, Chernoff $\mathrm{D}$, et al. Pretreatment multi-biomarker disease activity score and radiographic progression in early RA: results from the SWEFOT trial. Ann Rheum Dis. 2015;74(6):1102-9.

12. Rakieh C, Conaghan PG. Tofacitinib for treatment of rheumatoid arthritis. Adv Ther. 2013:30(8):713-26.

13. Fleischmann R, Cutolo M, Genovese MC, Lee EB, Kanik KS, Sadis S, et al. Phase Ilb dose-ranging study of the oral JAK inhibitor tofacitinib (CP$690,550)$ or adalimumab monotherapy versus placebo in patients with 
active rheumatoid arthritis with an inadequate response to diseasemodifying antirheumatic drugs. Arthritis Rheum. 2012;64(3):617-29.

14. Tanaka Y, Suzuki M, Nakamura H, Toyoizumi S, Zwillich SH, Tofacitinib SI. Phase II study of tofacitinib (CP-690,550) combined with methotrexate in patients with rheumatoid arthritis and an inadequate response to methotrexate. Arthritis Care Res (Hoboken). 2011;63(8):1150-8.

15. Burmester GR, Blanco R, Charles-Schoeman C, Wollenhaupt J, Zerbini C, Benda B, et al. Tofacitinib (CP-690,550) in combination with methotrexate in patients with active rheumatoid arthritis with an inadequate response to tumour necrosis factor inhibitors: a randomised phase 3 trial. Lancet (London, England). 2013;381(9865):451-60.

16. Fleischmann R, Kremer J, Cush J, Schulze-Koops H, Connell CA, Bradley JD, et al. Placebo-controlled trial of tofacitinib monotherapy in rheumatoid arthritis. N Engl J Med. 2012;367(6):495-507.

17. da Silva Chakr RM, Brenol JC, Behar M, Mendonca JA, Kohem CL, Monticielo $\mathrm{OA}$, et al. Is ultrasound a better target than clinical disease activity scores in rheumatoid arthritis with fibromyalgia? A case-control study. PLoS One. 2015;10(3):e0118620

18. Aletaha D, Neogi T, Silman AJ, Funovits J, Felson DT, Bingham CO, et al. 2010 rheumatoid arthritis classification criteria: an American College of Rheumatology/European league against rheumatism collaborative initiative. Arthritis Rheum. 2010;62(9):2569-81.

19. Choate EA, Kaeley GS, Brook J, Altman RD, FitzGerald JD, Floegel-Shetty AR, et al. Ultrasound detects synovitis in replaced and other surgically operated joints in rheumatoid arthritis patients. BMC Rheumatol. 2020;4:8.

20. Ranganath VK, Suliman YA, Brook J, Elashoff D, Ben-Artzi A, Olmos CP, et al. Musculoskeletal ultrasound in multi-center rheumatoid arthritis clinical trials: methodology for optimizing reliability of acquisition and real-time scoring [abstract]. Arthritis Rheum. 2015;67(suppl 10).

21. Hammer HB, Bolton-King P, Bakkeheim V, Berg TH, Sundt E, Kongtorp AK, et al. Examination of intra and interrater reliability with a new ultrasonographic reference atlas for scoring of synovitis in patients with rheumatoid arthritis. Ann Rheum Dis. 2011;70(11):1995-8.

22. Backhaus M, Ohrndorf S, Kellner H, Strunk J, Backhaus TM, Hartung W, et al. Evaluation of a novel 7-joint ultrasound score in daily rheumatologic practice: a pilot project. Arthritis Rheum. 2009;61(9):1194-201.

23. Chernoff D, Scott Eastman P, Hwang CC, Flake DD 2nd, Wang X, Kivitz A, et al. Determination of the minimally important difference (MID) in multibiomarker disease activity (MBDA) test scores: impact of diurnal and daily biomarker variation patterns on MBDA scores. Clin Rheumatol. 2019;38(2): 437-45.

24. Strand V, Lee EB, Yazici Y, Dikranian A, Wilkinson B, Takiya L, et al. Evaluation of disease activity in patients with rheumatoid arthritis treated with tofacitinib by RAPID3: post hoc analyses from two phase 3 trials. Clin Rheumatol. 2018;37(8):2043-53.

25. Naredo E, Moller I, Cruz A, Carmona L, Garrido J. Power Doppler ultrasonographic monitoring of response to anti-tumor necrosis factor therapy in patients with rheumatoid arthritis. Arthritis Rheum. 2008;58(8): 2248-56.

26. Sapundzhieva T, Karalilova R, Batalov A. Musculoskeletal ultrasound for predicting remission in patients with rheumatoid arthritis: results from a 1 year prospective study. Rheumatol Int. 2018;38(10):1891-99.

27. Han J, Geng Y, Deng X, Zhang Z. Subclinical synovitis assessed by ultrasound predicts flare and progressive bone erosion in rheumatoid arthritis patients with clinical remission: a systematic review and Metaanalysis. J Rheumatol. 2016;43(11):2010-8.

28. Ten Cate DF, Luime JJ, Swen N, Gerards AH, De Jager MH, Basoski NM, et al. Role of ultrasonography in diagnosing early rheumatoid arthritis and remission of rheumatoid arthritis--a systematic review of the literature. Arthritis Res Ther. 2013;15(1):R4.

29. Ellegaard K, Christensen R, Torp-Pedersen S, Terslev L, Holm CC, Konig MJ, et al. Ultrasound Doppler measurements predict success of treatment with anti-TNF-\&alpha; drug in patients with rheumatoid arthritis: a prospective cohort study. Rheumatology (Oxford). 2011;50(3):506-12.

30. Ranganath VK, Ben-Artzi A, Duffy E, Brook J, Taylor M, Choi S, et al. Elevated baseline power Doppler discriminates an RA subgroup highly responsive to therapy. Rheumatology (Oxford). 2015;54(12):2285-6.

31. Kawashiri SY, Nishino A, Shimizu T, Umeda M, Fukui S, Nakashima Y, et al. Ultrasound disease activity of bilateral wrist and finger joints at three months reflects the clinical response at six months of patients with rheumatoid arthritis treated with biologic disease-modifying anti-rheumatic drugs. Mod Rheumatol. 2017;27(2):252-6.

32. van der Helm-van Mil AH, Knevel R, Cavet G, Huizinga TW, Haney DJ. An evaluation of molecular and clinical remission in rheumatoid arthritis by assessing radiographic progression. Rheumatology (Oxford). 2013;52(5):839-46.

33. Curtis JR, Brahe $\mathrm{CH}$, Ostergaard M, Lund Hetland M, Hambardzumyan $\mathrm{K}$, Saevarsdottir $S$, et al. Predicting risk for radiographic damage in rheumatoid arthritis: comparative analysis of the multi-biomarker disease activity score and conventional measures of disease activity in multiple studies. Curr Med Res Opin. 2019;35(9):1483-93.

34. Brahe CH, Ostergaard M, Johansen JS, Defranoux N, Wang X, Bolce R, et al. Predictive value of a multi-biomarker disease activity score for clinical remission and radiographic progression in patients with early rheumatoid arthritis: a post-hoc study of the OPERA trial. Scand J Rheumatol. 2019;48(1): 9-16.

35. Curtis JR, Flake DD 2nd, Weinblatt ME, Shadick NA, Ostergaard M, Hetland $M L$, et al. Adjustment of the multi-biomarker disease activity score to account for age, sex and adiposity in patients with rheumatoid arthritis. Rheumatology (Oxford). 2019;58(5):874-83.

36. Hambardzumyan K, Saevarsdottir S, Forslind K, Petersson IF, Wallman JK Ernestam S, et al. A multi-biomarker disease activity score and the choice of second-line therapy in early rheumatoid arthritis after methotrexate failure. Arthritis Rheumatol. 2017;69(5):953-63.

37. van Vollenhoven RF, Bolce R, Hambardzumyan K, Saevarsdottir S, Forslind K, Petersson IF, et al. Brief report: enhancement of patient recruitment in rheumatoid arthritis clinical trials using a multi-biomarker disease activity score as an inclusion criterion. Arthritis Rheumatol. 2015;67(11):2855-60.

38. Edwards RR, Cahalan C, Mensing G, Smith M, Haythornthwaite JA. Pain, catastrophizing, and depression in the rheumatic diseases. Nat Rev Rheumatol. 2011;7(4):216-24.

\section{Publisher's Note}

Springer Nature remains neutral with regard to jurisdictional claims in published maps and institutional affiliations.

\section{Ready to submit your research? Choose BMC and benefit from:}

- fast, convenient online submission

- thorough peer review by experienced researchers in your field

- rapid publication on acceptance

- support for research data, including large and complex data types

- gold Open Access which fosters wider collaboration and increased citations

- maximum visibility for your research: over $100 \mathrm{M}$ website views per year

At BMC, research is always in progress.

Learn more biomedcentral.com/submissions 